\title{
A LIST OF STANDARD STAR DESIGNATION
}

\author{
F. Ochsenbein \\ C.D.S. Strasbourg \\ F. Spite \\ Observatoire de Paris Meudon
}

\begin{abstract}
Some rules about star designation have been edicted by Commission 5 of the IAU : they are briefly recalled and discussed. The essential rule, to be kept in mind by every author, is : when quoting a designation of a star, always quote the complete bibliographic reference of the paper defining this star designation. "Standard" designations can be an exception to this rule : it is proposed to publish in the CDS Bulletin a list of designations considered as "standard" ; the authors would be relieved of the obligation of quoting the references of these "standard" catalogues and could concentrate on the task of referencing the other catalogues.
\end{abstract}

\section{INTRODUCTION}

Several contributors to this Colloquium mentioned the problem and difficulties presently encountered with the designations of astronomical objects : these difficulties have been known for years. A discussion took place at the Grenoble meeting of IAU, in 1976. Working groups circulated proposals before the Montreal meeting of IAU (Jaschek and Pecker, 1979, Fernandez et al. 1979). From the discussion in Montreal, a few recommandations were extracted and adopted : let us concentrate here on stellar problem.

\section{VERY BRIGHT STARS}

The recommandation (Jaschek et al. 1980) precise that Beyer and Flamsteed designations are to be prefered for the brighter stars. Since we are involved in bibliographical work, we remark that, from an empirical point of view, some very bright stars are systematically quoted in the literature by their latin (or arabic) names such as : Vega, 
Sirius, Procyon, Arcturus, Pollux, Algol, Mira Ceti ... Since these designations are not ambiguous, we are ready to admit that these designations are acceptable if coupled with a second designations (number in a standard catalogue and/or coordinates). Here we encounter a principle, outlined by Prof. Jaschek (Jaschek et al., 1980) that a double designation is always extremely useful.

Commission 5 proposes Bayer designations. In our opinion, this should be considered with some caution : every astronomer who has ever done some proofreading of a paper including $v$ and $\nu, \chi$ and $k, \xi$ and $\zeta$ immediately knows what we mean. Moreover, there are some ambiguities with Bayer designations in the Southern Hemisphere (Hoffleit, 1979) Admittedly, most of the problems come from superscripts, and it could be decided to retain only the Bayer designations which are without superscripts. But Bayer designations used by differents authors do not agree about the presence of superscripts. Finally the solution should be : use only the Bayer notation exactly as they are defined in the next edition of the Yale Catalogue of Bright Stars = BS = HR. Note that you have to look carefully about this star in this catalogue for checking carefully the Bayer designation : then you have at hand the Bright Star number : please quote this number too as a second identifier, thus solving any ambiguity problem.

Dr Hoffleit (1979) recalls that it was recognised, a long time ago, that the use of latin lower case letters was to be avoided. Only a few variable stars have no other clearly defined name : it could be a good time now to progressively overcome this inconsistency.

Commission 5 then recommands the use of Flamsteed numbers; this designation has no real ambiguity problems. Let us note that one of the reasons for maintaining alive these older designations, is that they keep a link with observations in the past. May we remark that the limits on Constellations were changed by IAU, and that a few stars have officially lost their Flamsteed designation : in this case, the only way to maintain a link with past observations is to use more modern designations such as $\mathrm{HR}=\mathrm{BS}$ or $\mathrm{HD}$.

\section{BRIGHT STARS}

For stars a little fainter, Commission 5 recommands HR numbers : unfortunately, most people use BS instead for HR, and we must admit that for a young astronomer working in space physics, the logical 1 ink between a Bright Star number and the old Revision of Harvard Photometry is probably not evident.

\section{INTERMEDIATE STARS}

For intermediate stars, HD, BD, CD, CPD and Giclas numbers are con- 
venient. However, rather than G., GD, and GR, the replacement of G by the full name Giclas is recommanded, in order to avoid confusion with other (stellar and non-stellar) astronomical designations. Again, for all these stars, publication of coordinates as a second identifier would be useful, if cost (or policy) of publication allows it.

\section{FAINTER STARS}

Here, the situation is difficult : it is necessary to rely on special lists. It is recommanded to publish the coordinates, where available Moreover, it is necessary to quote the complete bibliographic references of these special list.

\section{ASTRONOMERS NAMES}

A few stars are quoted by the name of an astromer.

If the star has a "standard" designation, then always quote also the standard designation. If the star has no "standard" designation, always quote the complete bibliographic reference of the paper identifying the star, and the coordinates.

We would not have insisted on this very small sample of stars, if errors were not often repeated in the modern literature. For instance, a book published in 1976 repeats the false statement that Barnard's star would be identical to $\mathrm{BD} 4^{\circ} 3561$, in spite of continuous efforts of Prof. Gliese to point out this error.

A 1 ist of stars designated by astronomers'names will be published in a forthcoming CDS Information Bulletin.

\section{VARIABLE STARS}

The nomenclature of these stars is kept up to date by our colleagus in Moscow. We mentioned hereabove that probably lower case latin letters should progressively be replaced (in order to avoid errors due to misprints).

\section{STARS IN CLUSTERS, IN MAGELLANIC CLOUDS}

Nothing was proposed. For stars in clusters, Mermilliod (1977) proposed a special numeric code; the IAU Commission of clusters edicted a nomenclature rule for clusters, materialized by the Catalogue of Lynga (1980) : a solution could probably be found in the near future. 


\section{CONCLUSION}

The solution to be adopted, in order to completely clarify the nomenclature problems, are not easy to find. However three simple rules, if followed by all authors, could considerably alleviate the problems :

1.1. The simultaneous use of two designations.

1.2. The systematic citation of the complete bibliographic references of the designations (only the designation included in an accepted list of "standard" designations could be excepted).

1.3. The systematic indication of the equinox of the coordinates.

Let us hope that al1 the participants in this Colloquium will at least remember these three rules, follow them and teach them to their students and younger colleagues; this would be a first step in bringing astronomical nomenclature to an orderly state, not spoiling any more the prestige of Astronomy.

\section{FINAL MESSAGE}

Compilations of stars, built up by specialists, are very important and very useful work. This is so important for bibliographical work and nomenclature problems, that anybody aware of a compilation in progress somewhere should inform immediately the authors of this communication.

\section{REFERENCES}

Fernandez A., Lortet M.C., Spite F. : 1979,"Compilation of Data for preparing resolutions about nomenclature rules", unpublished.

Hoffleit D. : 1979, Bull. Inform. CDS, 17, pp. 38-65

Jaschek C., Ochsenbein F. and Bidelman W.P. : 1980, Bu11. Inform. CDS 18, pp 41-50

Jascbek C., Pecker J.C. : 1979, Bu11. Inform. CDS 16, pp. 57-70

Lyngå G, Lundstrom I. : 1980, in IAU Symp. 85, 123 (D. Reidel Pub1. Co., Dordrecht, Ho11 and)

Mermilliod J.C. : 1977, "Principles of a Coded numbering System and its application to open clusters, in Compilation Critical Evaluation and Distribution of Stellar Data!' IAU coll. 35, C. Jaschek and G.A. Wilkins, Eds, D. Reidel Publ. Co., Dordrecht, Holland. 